nephron

Practice
Nephron 2016;133:183-192

DOI: $10.1159 / 000447601$
Received: January 5, 2016

Accepted after revision: June 10, 2016

Published online: July 1, 2016

\title{
Non-Contrast Renal Magnetic Resonance Imaging to Assess Perfusion and Corticomedullary Differentiation in Health and Chronic Kidney Disease
}

\author{
Keith A. Gillis ${ }^{a}$ Christie McComb $^{a}$ Rajan K. Patel ${ }^{a}$ Kathryn K. Stevens ${ }^{a}$ \\ Markus P. Schneider ${ }^{d}$ Aleksandra Radjenovic ${ }^{a}$ Scott T.W. Morris ${ }^{b}$ \\ Giles H. Roditic Christian Delles ${ }^{\mathrm{a}}$ Patrick B. Mark ${ }^{\mathrm{a}}$ \\ ${ }^{a}$ Institute of Cardiovascular and Medical Sciences, University of Glasgow, ${ }^{b}$ Glasgow Renal and Transplant Unit, Queen \\ Elizabeth University Hospital, and 'Department of Radiology, Glasgow Royal Infirmary, Glasgow, UK; ${ }^{\mathrm{d}}$ Department of \\ Nephrology and Hypertension, University of Erlangen-Nuremberg, Erlangen-Nuremberg, Germany
}

\section{Key Words}

Magnetic resonance imaging $\cdot$ Arterial spin labeling $\cdot$ Renal perfusion · Chronic kidney disease

\footnotetext{
Abstract

Aims: Arterial spin labelling (ASL) MRI measures perfusion without administration of contrast agent. While ASL has been validated in animals and healthy volunteers (HVs), application to chronic kidney disease (CKD) has been limited. We investigated the utility of ASL MRI in patients with CKD. Methods: We studied renal perfusion in $24 \mathrm{HVs}$ and 17 patients with CKD (age 22-77 years, 40\% male) using ASL MRI at 3.0T. Kidney function was determined using estimated glomerular filtration rate (eGFR). T1 relaxation time was measured using modified look-locker inversion and flowsensitive alternating inversion recovery true-fast imaging and steady precession was performed to measure cortical
}

and whole kidney perfusion. Results: T1 was higher in CKD within cortex and whole kidney, and there was association between T1 time and eGFR. No association was seen between kidney size and volume and either T1, or ASL perfusion. Perfusion was lower in CKD in cortex (136 \pm 37 vs. $279 \pm$ $69 \mathrm{ml} / \mathrm{min} / 100 \mathrm{~g} ; \mathrm{p}<0.001)$ and whole kidney $(146 \pm 24 \mathrm{vs}$. $221 \pm 38 \mathrm{ml} / \mathrm{min} / 100 \mathrm{~g} ; \mathrm{p}<0.001)$. There was significant, negative, association between $\mathrm{T} 1$ longitudinal relaxation time and ASL perfusion in both the cortex $(r=-0.75, p<$ $0.001)$ and whole kidney $(r=-0.50, p<0.001)$. There was correlation between eGFR and both cortical $(r=0.73, p<0.01)$ and whole kidney $(r=0.69, p<0.01)$ perfusion. Conclusions: Significant differences in renal structure and function were demonstrated using ASL MRI. T1 may be representative of structural changes associated with CKD; however, further investigation is required into the pathological correlates of reduced ASL perfusion and increased T1 time in CKD.

(c) 2016 S. Karger AG, Basel

\section{KARGER}

E-Mail karger@karger.com

www.karger.com/nef
(C) 2016 S. Karger AG, Basel

$1660-8151 / 16 / 1333-0183 \$ 39.50 / 0$
Dr. Keith A. Gillis

Institute of Cardiovascular and Medical Sciences

University of Glasgow, 126 University Place

Glasgow G12 8TA (UK)

E-Mail keithgillis@ nhs.net 


\section{Introduction}

Renal perfusion is an important physiological parameter in health and disease. In normal physiology, renal blood flow is an important determinant of oxygen supply and glomerular filtration rate (GFR) [1], while in chronic kidney disease (CKD), renal microvascular dysfunction is one of a number of common pathological mechanisms involved in the progression of disease, irrespective of the initiating insult.

Despite this crucial role of perfusion in renal physiology and disease, in vivo measurement remains a challenge in both clinical and research settings, as established methods are associated with a number of inherent drawbacks. Measurement of the clearance of para-aminohippuric acid (PAH) is time consuming and invasive [2], while $\mathrm{CT}$ and nuclear medicine techniques carry a radiation burden, with the former requiring the administration of nephrotoxic iodinated contrast. Dynamic contrast enhanced (DCE) MR techniques can be used to measure renal perfusion, but the administration of gadoliniumbased agents is now relatively contraindicated in patients with renal impairment due to an association with nephrogenic systemic fibrosis [3].

Arterial spin labelling (ASL) MRI is an imaging technique allowing non-invasive measurement of renal perfusion using magnetically labelled blood as a contrast agent. Protons in blood are labelled by application of a saturation, or inversion, radiofrequency pulse, which then alter tissue magnetization upon exchange with blood within capillary beds. An unlabelled image is also acquired, and the ASL signal is determined by subtraction of one image from the other. ASL MRI has an inherently low signal-to-noise ratio, due to the low contribution of inflowing blood to total tissue magnetisation. Nevertheless, ASL MRI has been validated in animals against a microsphere technique [4], and in an explanted kidney model [5], with close correlation observed between methods. In humans, good reproducibility has been confirmed in healthy volunteer (HV) studies [6]. For example, in a recent study, a coefficient of variance of 9.2 and $7.1 \%$ for cortical perfusion and whole kidney perfusion was demonstrated [7]. A small number of studies have shown reduced perfusion in patients with CKD compared to controls $[8,9]$, and in poorly functioning kidney transplants compared to transplants with better function [10-12].

Nevertheless, ASL MRI has not yet entered widespread clinical use, hampered by lack of standardization in sequence acquisition protocols, and post-processing methods. The utility of ASL MRI as a marker for disease sever- ity and progression in $\mathrm{CKD}$, and as a measure of response to therapy, is yet to be determined. We therefore investigated the use of ASL MRI for the assessment of patients with CKD.

\section{Materials and Methods}

Patients with CKD were recruited from the general nephrology clinic at the Glasgow Renal and Transplant Unit, while HVs were recruited via local advertisement. Subjects attended on a single occasion, undergoing clinical and biochemical assessment, and subsequent MRI. All subjects gave written informed consent and the local ethics committee approved the study. The study is registered with a clinical trials database (ISRCTN 12301736) and was carried out in compliance with the Declaration of Helsinki.

\section{Biochemical Measurements}

Baseline serum biochemistry and haematology measurements and urinary protein and creatinine quantification were obtained. Estimated GFR (eGFR) was calculated from the measured serum creatinine using the CKD Epidemiology Collaboration (CKDEPI) formula [13]. Proteinuria was measured using a spot protein to creatinine ratio (PCR) from a random urine sample.

\section{Magnetic Resonance Imaging}

MRI was performed on a Siemens Magnetom Verio 3.0T scanner (Siemens Erlangen, Germany), using a 6-channel phased array body coil. A half Fourier acquisition single shot turbo spin echo localizer sequence was used to identify the location of the kidneys and vessels, using the following parameters: TR 1,400 ms, TE 93 $\mathrm{ms}$, voxel size $2.1 \times 1.5 \times 5 \mathrm{~mm}^{3}$, refocusing pulse flip angle $160^{\circ}$, number of slices 30, turbo factor 179, bandwidth $781 \mathrm{~Hz} /$ pixel. ASL was performed using a flow-sensitive alternating inversion recovery (FAIR) perfusion preparation with true fast imaging and steady precession (True-FISP) acquisition. A single sagittal double oblique slice of both kidneys was obtained, positioned at the midpoint of each axis, moved posteriorly to avoid major vessels. Three images with alternating selective and non-selective inversions were obtained in a single acquisition during a $12 \mathrm{~s}$ breath-hold, and this was repeated 5 times. In addition, an image with no ASL preparation was acquired to measure equilibrium magnetization. FAIR True-FISP parameters were: inversion time (TI) $900 \mathrm{~ms}$, repetition time $3.65 \mathrm{~ms}$, echo time $1.83 \mathrm{~ms}$, flip angle $60^{\circ}$, field of view $380 \times$ $380 \mathrm{~mm}$, in plane resolution $2.0 \times 1.5 \mathrm{~mm}^{2}$, matrix size $256 \times 192$, and slice thickness $10 \mathrm{~mm}$. T1 was acquired during a separate breath-hold by a modified look-locker inversion recovery sequence, with the following parameters: TR $740 \mathrm{~ms}$, TE $1.1 \mathrm{~ms}$, voxel size $2.0 \times 1.5 \times 6 \mathrm{~mm}^{3}$, flip angle $35^{\circ}$, starting TI $125 \mathrm{~ms}$, TI increment $80 \mathrm{~ms}$, number of inversions 3, bandwidth $930 \mathrm{~Hz} /$ pixel. T1 was computed pixel wise using a non-linear curve fitting algorithm, using the three-parameter signal model [14]. The total scan time was approximately $15 \mathrm{~min}$.

\section{Image Analysis}

Renal anatomy was assessed on localizer images using a commercially available multi-modality post-processing workstation (Siemens Syngo, Siemens, Erlangen, Germany). Kidney length was 
measured on coronal images and volume was measured using a voxel count method by tracing contours on each slice of a 22 slice transverse oriented image volume. T1 time was measured in cortex, medulla, and whole kidney, and corticomedullary differentiation (CMD) was calculated as the ratio of cortex to medulla T1 time. Post processing was performed using in-house software (MATLAB 8.4 R2014b; MathWorks, Natick, Mass., USA). An averaged ASL subtraction image was produced from registered subtraction images derived from each breath-hold. This was fitted to the M0 and T1 data using the standard ASL kinetic model [15] to produce a perfusion map. Image co-registration of ASL, M0 and T1 maps was performed using an enhanced correlation coefficient maximization algorithm with affine transformations [16]. Pixelwise computation of perfusion was performed according to the following formula, where $\mathrm{f}=$ perfusion, $\Lambda=$ tissue-blood partition coefficient $(0.8 \mathrm{ml} / \mathrm{g}$ in kidney), $\mathrm{M} 0=$ equilibrium magnetisation, $\Delta \mathrm{M}=\mathrm{ASL}$ signal, $\mathrm{T} 1$ = longitudinal relaxation time, $\mathrm{TI}=$ inversion time:

$$
\mathrm{f}=\frac{\lambda}{2 \mathrm{TI}} \frac{\Delta \mathrm{M}(\mathrm{TI})}{\mathrm{M}_{0}} \exp \frac{\mathrm{TI}}{\mathrm{T} 1}
$$

Regions of interest (ROIs) were drawn onto the perfusion map to measure cortical and whole kidney perfusion. Total single kidney perfusion was calculated by multiplying the renal perfusion normalised per gram of renal tissue by the renal mass, assuming that the mass is $1 \mathrm{~g}$ per $1 \mathrm{~cm}^{3}$ of renal tissue. For each individual, the total kidney perfusion analogous to the renal blood flow was calculated by combining the kidney blood flow of the left and right kidneys. A single operator performed image analysis.

\section{Statistical Analysis}

Results are expressed as mean \pm SD. T1 time and perfusion were measured in cortical and whole kidney ROIs, and ASL measurements are expressed by unit of mass (100 g), which is typical in the standard kinetic model. Between group differences in T1 time, CMD and perfusion were evaluated using independent samples Student's t tests. Evaluation of correlation between MRI measurements and serum and urine biochemistry parameters was performed using the Pearson's correlation coefficient. Throughout, $\mathrm{p}$ values $<0.05$ were deemed significant. Data were analysed using IBM SPSS Statistics version 22.0 (IBM, Armonk, New York, N.Y., USA).

\section{Results}

\section{Baseline Data}

Twenty four HV and 17 patients with CKD were recruited; the demographic data for each group is displayed in table 1 . The CKD group was significantly older $(\mathrm{p}<$ $0.05)$, and had higher blood pressure $(\mathrm{p}<0.05)$. CKD-EPI eGFR was $39.8 \pm 25.2 \mathrm{ml} / \mathrm{min} / 1.73 \mathrm{~m}^{2}$ in the CKD group and $99.6 \pm 14.0 \mathrm{ml} / \mathrm{min} / 1.73 \mathrm{~m}^{2}$ in the $\mathrm{HV}$ group $(\mathrm{p}<$ $0.001)$.

Non-Contrast Renal MRI in Health and Disease
Table 1. Baseline parameters. Baseline clinical and biochemical parameters are shown of HVs and patients with CKD

\begin{tabular}{lccr}
\hline & HVs & CKD & p value \\
\hline Number & 24 & 17 & \\
Age, years & $47 \pm 14$ & $56 \pm 10$ & $<0.05$ \\
Body mass index, kg/m ${ }^{2}$ & $26.5 \pm 5.3$ & $29.3 \pm 3.4$ & 0.06 \\
Blood pressure, mm Hg & $132 / 83 \pm 15 / 8$ & $151 / 90 \pm 26 / 14$ & $<0.05$ \\
Mean arterial blood & & & \\
$\quad$ pressure, mm Hg & $99 \pm 9$ & $110 \pm 17$ & $<0.05$ \\
CKD-EPI eGFR, ml/ & & & \\
$\quad$ min/1.73 m ${ }^{2}$ & $99.6 \pm 14.0$ & $39.9 \pm 25.2$ & $<0.001$ \\
Serum creatinine, $\mu$ mol/1 & $68 \pm 10$ & $184 \pm 69$ & $<0.001$ \\
Primary renal diagnosis, $\mathrm{n}$ & & & \\
$\quad$ Diabetes & & 2 & \\
Glomerulonephritis & & 8 & \\
$\quad$ Renovascular disease & & 4 & \\
Other & & 2 & \\
CKD-cause unknown & & 1 & \\
CKD stage, $\mathrm{n}$ & & & \\
1 & & 2 & \\
2 & & 1 & \\
3 & & 4 & \\
4 & & 0 & \\
5 & & & \\
\hline
\end{tabular}

Results are shown as mean \pm SD.

Table 2. MRI parameters. Measurements of renal anatomy, T1 longitudinal relaxation time, and ASL MRI perfusion are shown in HVs and CKD

\begin{tabular}{|c|c|c|c|}
\hline Parameter & $\mathrm{HVs}$ & CKD & $\mathrm{p}$ value \\
\hline Kidney length, cm & $10.5 \pm 0.8$ & $9.7 \pm 0.9$ & $<0.05$ \\
\hline Kidney volume, $\mathrm{cm}^{3}$ & $167.1 \pm 35.0$ & $160.1 \pm 53.4$ & 0.62 \\
\hline Cortical T1 time, ms & $1,366 \pm 122$ & $1,529 \pm 77$ & $<0.001$ \\
\hline Whole kidney $\mathrm{T} 1$ time, $\mathrm{ms}$ & $1,472 \pm 91$ & $1,550 \pm 81$ & $<0.01$ \\
\hline CMD & $0.84 \pm 0.07$ & $0.94 \pm 0.07$ & $<0.001$ \\
\hline $\begin{array}{l}\text { Mean cortical perfusion, } \mathrm{ml} / \\
\mathrm{min} / 100 \mathrm{~g}\end{array}$ & $279 \pm 69$ & $136 \pm 37$ & $<0.001$ \\
\hline $\begin{array}{l}\text { Mean whole kidney perfusion, } \\
\mathrm{ml} / \mathrm{min} / 100 \mathrm{~g}\end{array}$ & 221 & 146 & $<0.001$ \\
\hline Mean kidney perfusion, $\mathrm{ml} / \mathrm{min}$ & $366 \pm 79$ & $223 \pm 75$ & $<0.001$ \\
\hline Total renal perfusion, $\mathrm{ml} / \mathrm{min}$ & $731 \pm 159$ & $446 \pm 150$ & $<0.001$ \\
\hline
\end{tabular}

Results are shown as mean $\pm \mathrm{SD}$.

\section{Renal Anatomy}

Renal anatomical data is shown in table 2. Kidney length was significantly shorter in the CKD group compared with the HV group ( $\mathrm{p}<0.05$ ); however, renal volume was no different between the 2 . The CKD group had 


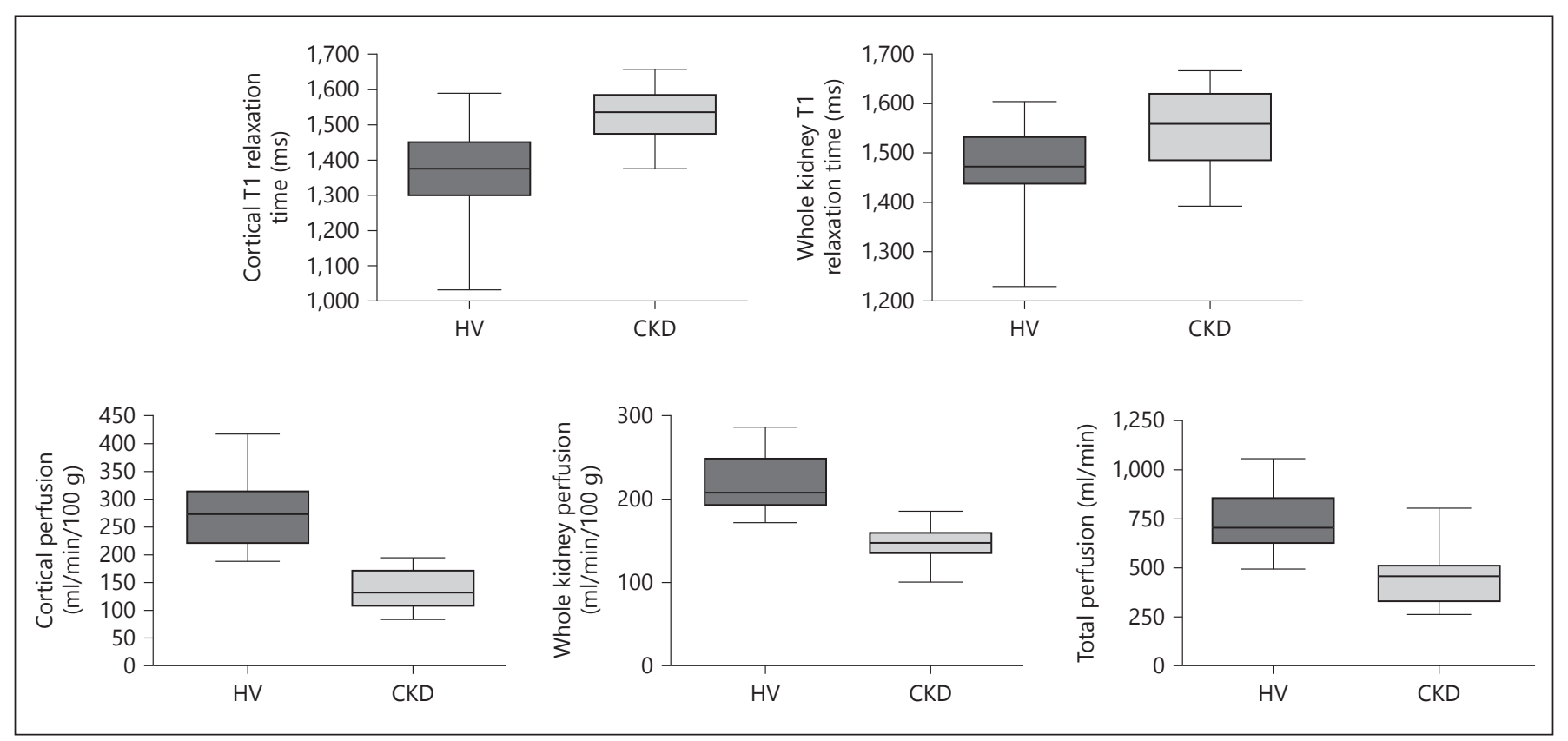

Fig. 1. Box and whisker plot of T1 longitudinal relaxation time and perfusion in HVs and CKD.

significantly higher T1 longitudinal relaxation time both measured in the cortex $(\mathrm{p}<0.001)$ and the whole kidney $(\mathrm{p}<0.01)$ ROI (fig. 1). Furthermore, CMD was significantly higher in CKD than in HV $(\mathrm{p}<0.001)$.

\section{Renal Perfusion}

Renal perfusion was significantly lower in the CKD group (table 2; fig. 1). In the CKD cohort, mean cortical perfusion was $136 \pm 37 \mathrm{ml} / \mathrm{min} / 100 \mathrm{~g}$ in comparison to $279 \pm 69 \mathrm{ml} / \mathrm{min} / 100 \mathrm{~g}$ in the HV cohort $(\mathrm{p}<0.001)$. Similarly, whole kidney perfusion was reduced in the CKD group at $146 \pm 24 \mathrm{ml} / \mathrm{min} / 100 \mathrm{~g}$ compared to $221 \pm 38 \mathrm{ml} /$ $\mathrm{min} / 100 \mathrm{~g}(\mathrm{p}<0.001)$. Furthermore, total renal perfusion was $446 \pm 150 \mathrm{ml} / \mathrm{min}$ in CKD compared to $731 \pm 158 \mathrm{ml} /$ min in HV ( $p<0.001)$. Typical perfusion maps from both groups are shown in figure 2.

\section{Intra-Observer Variability}

Intra-observer variation of cortical perfusion measurements was $7.3 \%$ with intra-class correlation (ICC) of 0.98 , while variation of whole kidney perfusion measurements was found to be $4.4 \%$ with ICC of 0.96 .

\section{Association between Renal Anatomical and \\ Functional Parameters}

There was significant, negative, association between T1 longitudinal relaxation time and ASL perfusion mea- sured in both the cortex $(\mathrm{r}=-0.75, \mathrm{p}<0.001)$ and whole kidney $(\mathrm{r}=-0.50, \mathrm{p}<0.001)$. No significant association was seen between kidney length or volume and either ASL perfusion measurements, or T1 longitudinal relaxation time.

\section{Correlation of Clinical, Biochemical and MRI \\ Parameters}

Both cortical and whole kidney perfusion were found to have a negative association with age (respectively, $\mathrm{r}=$ $-0.48, \mathrm{p}<0.01 ; \mathrm{r}=-0.51, \mathrm{p}<0.01)$. While there was no association between blood pressure and cortical perfusion, a negative correlation was observed between whole kidney perfusion and mean arterial blood pressure $(\mathrm{r}=$ $-0.33, \mathrm{p}<0.05)$.

Correlation was seen between eGFR and both whole kidney T1 longitudinal relaxation time $(\mathrm{r}=-0.40, \mathrm{p}<$ $0.05)$ and cortical T1 time $(\mathrm{r}=-0.58, \mathrm{p}<0.001)$. Furthermore, significant correlation was seen between eGFR and both cortical perfusion $(\mathrm{r}=0.73, \mathrm{p}<0.01)$ and whole kidney perfusion $(\mathrm{r}=0.69, \mathrm{p}<0.01)$. There was also significant correlation between total renal perfusion and eGFR $(\mathrm{r}=0.69, \mathrm{p}<0.01)$. PCR was negatively correlated with both cortical $(\mathrm{r}=-0.60, \mathrm{p}<$ $0.01)$ and whole kidney perfusion $(\mathrm{r}=-0.43, \mathrm{p}<0.05$; fig. 3). 


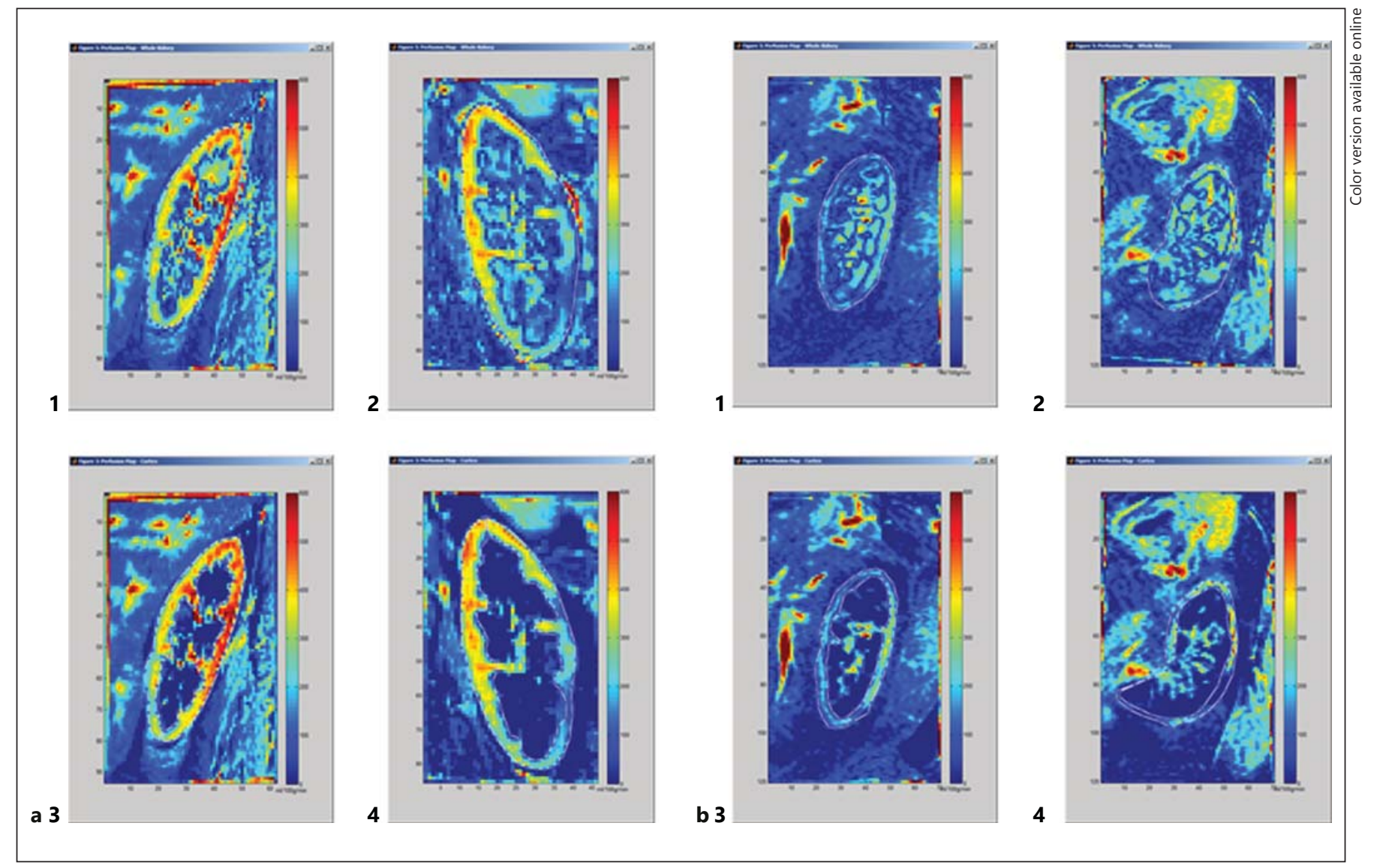

Fig. 2. ASL MRI perfusion maps from a $H V$ (a) and patient with CKD stage $3 / 4$ (b) with an eGFR of $30 \mathrm{ml} /$ $\mathrm{min} / 1.73 \mathrm{~m}^{2}$. Both whole kidney (1 and 2) and cortical (3 and 4) perfusion are demonstrated. Cortical thinning, reduced $\mathrm{CMD}$, and reduced global perfusion can be seen in CKD.
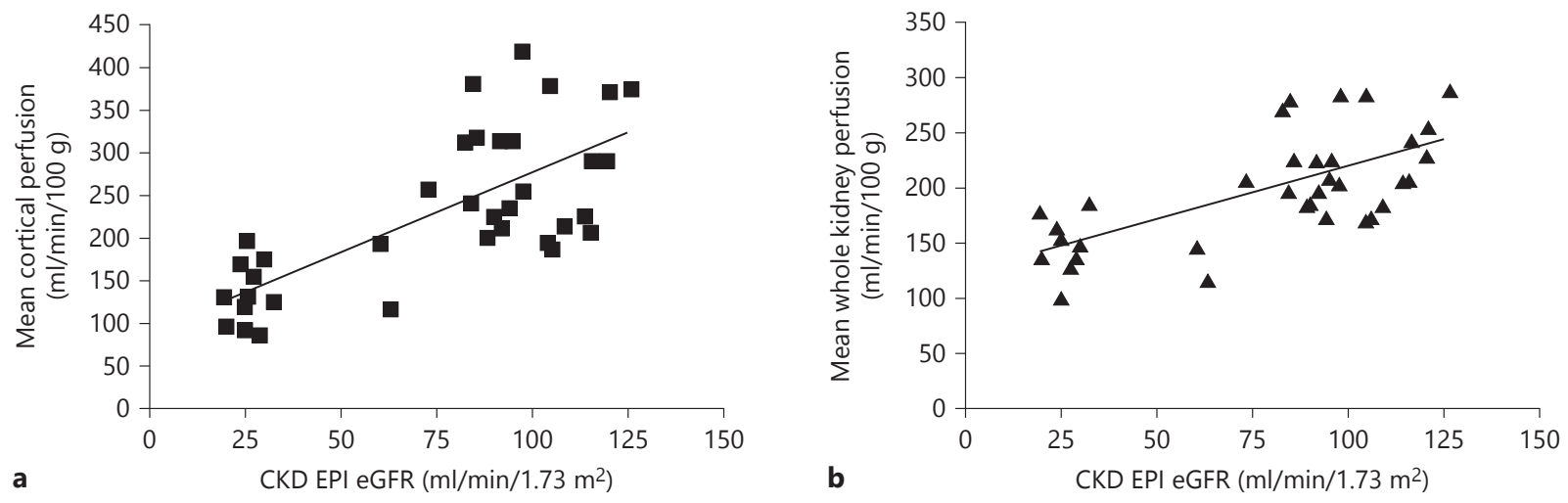

Fig. 3. Association between biochemical measurements and ASL. Correlation was observed between eGFR and cortical perfusion $(\mathrm{r}=0.73, \mathrm{p}<0.01 ; \mathbf{a})$, and eGFR and whole kidney perfusion $(\mathrm{r}=0.69, \mathrm{p}<0.01 ; \mathbf{b})$. 


\section{Discussion}

CKD has a tendency to worsen despite treatment of blood pressure and any other reversible or aetiological factors, and there is evidence that common pathological mechanisms are responsible for this irrespective of the original renal insult. Renal damage has been shown to correlate primarily with tubulointerstitial injury [17], characterised by a vicious cycle of microvasculature dysfunction leading to tubular atrophy and fibrosis [18]. In vivo biomarkers to assess renal progression are lacking, and emerging techniques such as ASL MRI may provide the much needed insight into renal perfusion and thus extent of renal damage.

We found that cortical perfusion is reduced from 279 $\mathrm{ml} / \mathrm{min} / 100 \mathrm{~g}$ in $\mathrm{HV}$ to $136 \mathrm{ml} / \mathrm{min} / 100 \mathrm{~g}$ in patients with $\mathrm{CKD}$, with correlation between perfusion and degree of renal impairment quantified by eGFR. Whole kidney perfusion is similarly reduced from 221 to $146 \mathrm{ml} / \mathrm{min} / 100 \mathrm{~g}$. This is in keeping with previous measurements of renal perfusion in health and disease, and the finding of reduced native kidney perfusion in CKD has also previously been demonstrated $[9,12]$. While our perfusion values are lower than those found in other studies, this CKD cohort represents the largest to undergo ASL MRI and included patients with more advanced renal impairment than previously studied. Our findings demonstrate strong correlation of renal function to perfusion across a broad range of CKD-EPI eGFR, ranging from 20 to $126 \mathrm{ml} /$ $\mathrm{min} / 1.73 \mathrm{~m}^{2}$.

Previous human studies using ASL MRI are summarised in table 3 , which demonstrate the range of perfusion values previously demonstrated. The broad range could be ascribed to differences in ASL sequence, imaging strategy, and post processing as well as true differences in study population. For example, different strategies have been employed to circumvent the problem of renal respiratory motion, including breath-holding, respiratory gating, or post processing registration. Gardener and Francis [19] found no difference in perfusion measurements made with either breath-holding or free breathing, but found reduced perfusion when background suppression was used to improve image quality, showing that some variations in imaging approach cause differences in perfusion measurements. Our ASL technique resulted in a scan time of $15 \mathrm{~min}$, and breathholding time of $12 \mathrm{~s}$, which was tolerated by all participants.

ASL has been validated in animal models using microsphere techniques and using explanted organs undergo- ing haemoperfusion. In normal renal function, strong correlation between ASL and both DCE MRI perfusion [20], and PAH clearance [21] has been shown. Validation of ASL against a gold standard perfusion technique has not, to our knowledge, been undertaken in a CKD population. Given that quantitative measurement of perfusion using the standard ASL kinetic model is dependent on T1 time, it is possible that structural changes in CKD are at least partly responsible for the functional changes measured by ASL MRI. In keeping with previous studies [22], we have shown that $\mathrm{T} 1$ time is significantly higher in $\mathrm{CKD}$, and that $\mathrm{T} 1$ time shows strong correlation with CKD-EPI eGFR. Lee et al. [23] previously showed that cortical T1, but not medullary T1 time showed strong correlation with single kidney GFR measured by renography. These differences may be accounted for by changes in extracellular composition, fibrosis, or in the microvasculature, and further investigation is required to understand the association between the pathological changes in CKD, T1 time, and ASL perfusion. Notably, there was stronger association between eGFR and whole kidney ASL perfusion $(r=0.69, \mathrm{p}<0.01)$ than $\mathrm{T} 1$ time $(\mathrm{r}=0.40, \mathrm{p}<0.05)$, suggesting that ASL does grant some additional information into renal physiology in CKD, in addition to the structural changes identified by differences in T1 time. Additionally, there was no association between ASL and kidney size or volume, suggesting that the difference in perfusion in CKD is not entirely attributable to tissue atrophy.

ASL MRI is but one of a number of emerging MRI techniques, which may have utility in $\mathrm{CKD}$, such as blood oxygen level dependent imaging, and diffusion weighted and diffusion tensor imaging. Future research should be guided at identifying the imaging correlates of renal fibrosis in CKD, as this may allow the identification of biomarkers, which can prognosticate, guide therapy, and act as surrogate markers of renal progression in studies of novel therapeutics in CKD.

Our study has a number of limitations. Our CKD cohort has a variety of renal pathologies and while common pathological mechanisms underpin all chronic kidney disorders, it is possible that perfusion abnormalities may predominate in certain aetiologies of CKD over others. Despite attempts to match the 2 groups, mean age was higher in the CKD than HV cohort, and therefore the changes in ASL MRI may not be independent of aging. Despite being one of the largest ASL studies in CKD, even larger studies are required to confirm our findings and exclude the possibility of group effects confounding some of the associations with the measured biochemical 


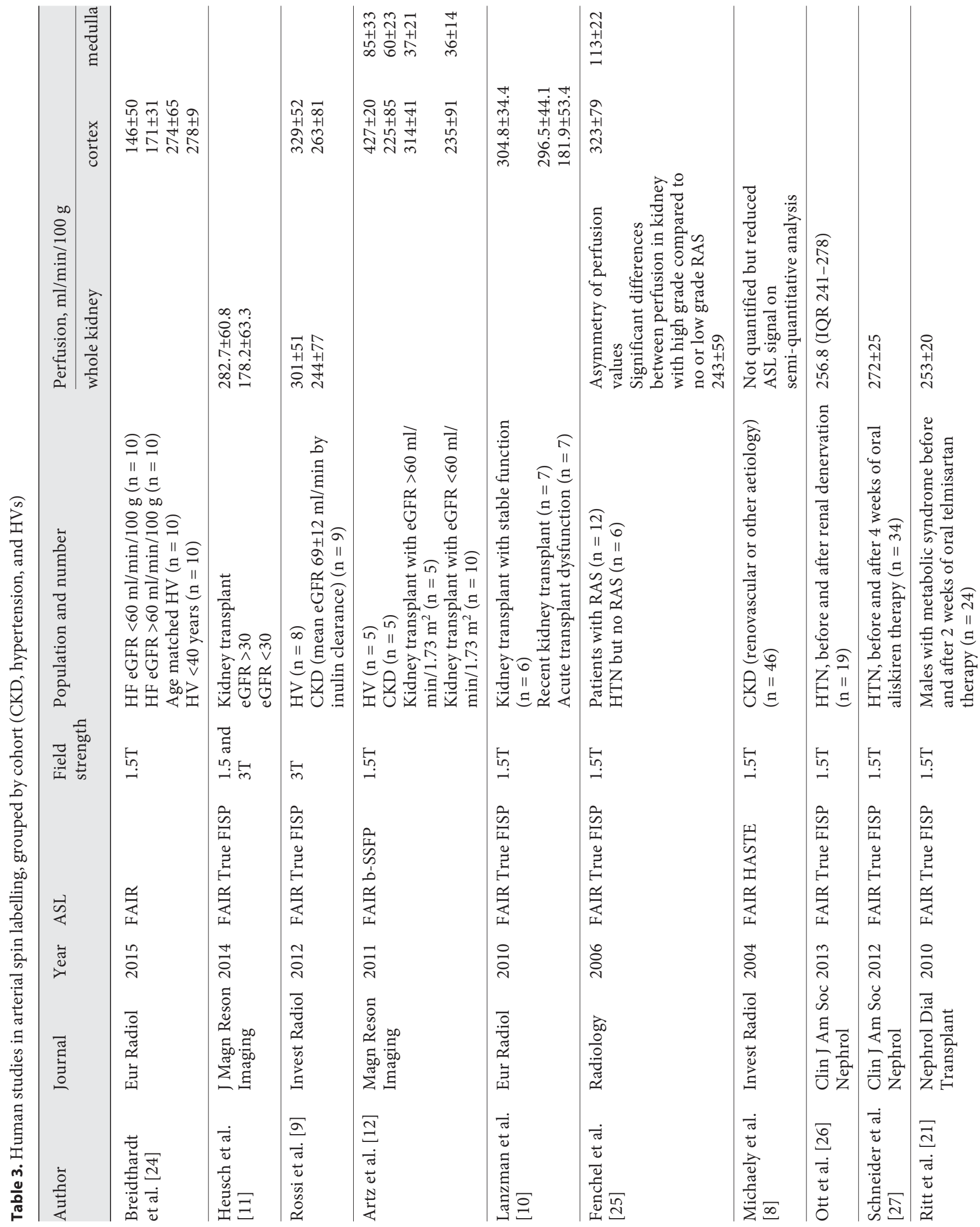




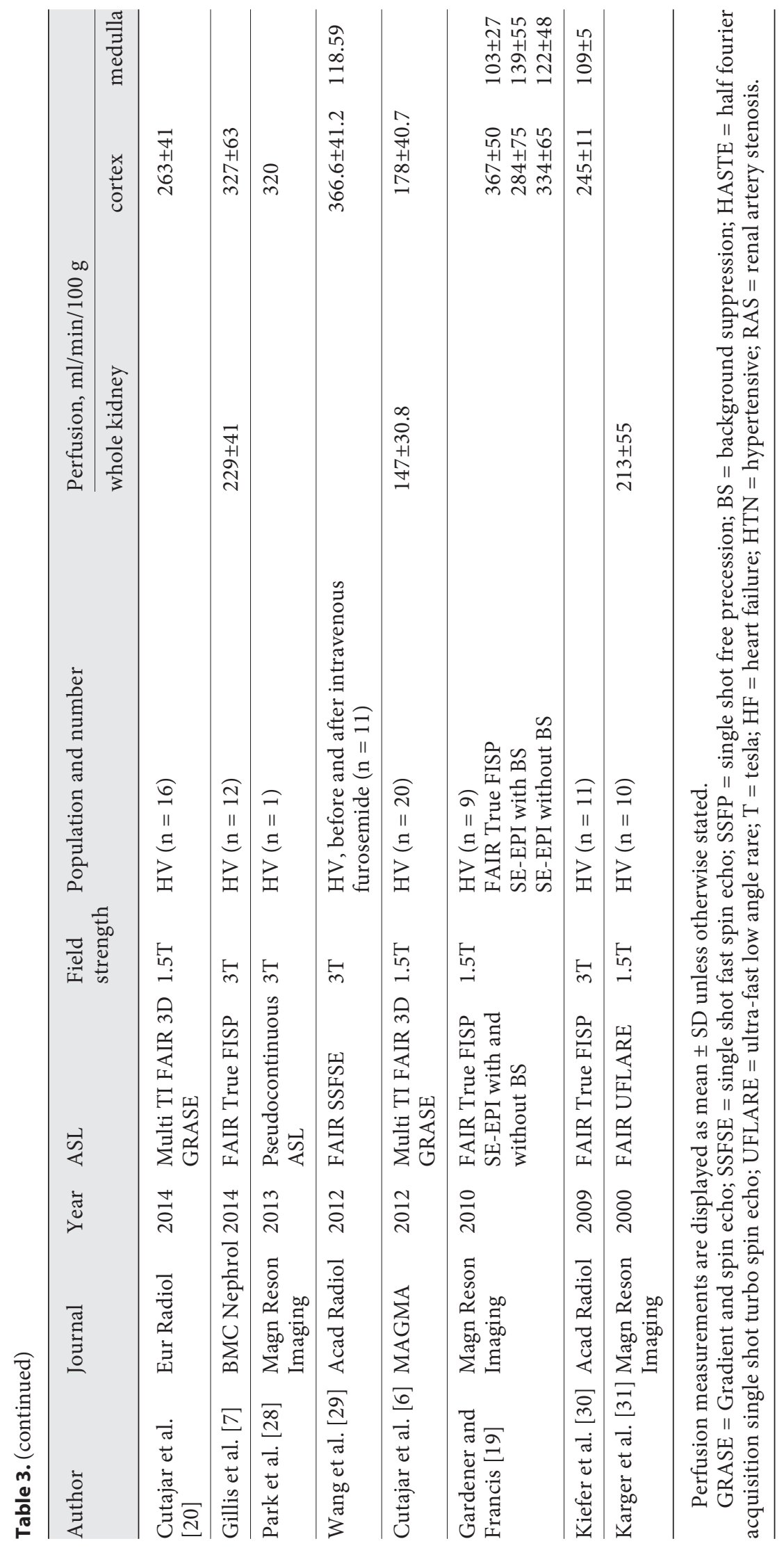


parameters. Furthermore, we have used the standard ASL kinetic model, which is primarily validated in healthy volunteers and assumes constant arterial transit time and blood tissue exchange. Differences in these factors may artefactually alter perfusion measurements in $\mathrm{CKD}$, and as previously discussed, further research is necessary to validate the use of ASL in the CKD population. Lastly, our study was carried out using 3.0T MRI, which is in general less available in clinical use and further work will be required to translate our findings to $1.5 \mathrm{~T}$ platform, as it is more commonly used in clinical practice.

In conclusion, we have shown significant differences in renal perfusion measured with ASL MRI in a group of patients with advanced CKD, and shown correlation to renal parameters such as eGFR.

\section{Acknowledgements}

This study was funded by Darlinda's Charity for renal research. We would also like to thank Peter Weale and Patrick Revell (Siemens Healthcare, UK) for provision of a work-in-progress ASL sequence and for their ongoing support.

\section{Disclosure Statement}

There are no conflicts of interest to declare.

\section{Statement of Ethics}

This study was approved by the local ethics committee and was conducted according to the Declaration of Helsinki. All participants gave written informed consent.

\section{References}

1 Evans RG, Ince C, Joles JA, Smith DW, May CN, O'Connor PM, Gardiner BS: Haemodynamic influences on kidney oxygenation: clinical implications of integrative physiology. Clin Exp Pharmacol Physiol 2013;40:106122.

-2 Cole BR, Giangiacomo J, Ingelfinger JR, Robson AM: Measurement of renal function without urine collection. A critical evaluation of the constant-infusion technic for determination of inulin and para-aminohippurate. $\mathrm{N}$ Engl J Med 1972;287:1109-1114.

-3 Collidge TA, Thomson PC, Mark PB, Traynor JP, Jardine AG, Morris ST, Simpson K, Roditi GH: Gadolinium-enhanced MR imaging and nephrogenic systemic fibrosis: retrospective study of a renal replacement therapy cohort. Radiology 2007;245:168-175.

-4 Artz NS, Wentland AL, Sadowski EA, Djamali A, Grist TM, Seo S, Fain SB: Comparing kidney perfusion using noncontrast arterial spin labeling MRI and microsphere methods in an interventional swine model. Invest Radiol 2011;46:124-131.

5 Warmuth C, Nagel S, Hegemann O, Wlodarczyk W, Lüdemann L: Accuracy of blood flow values determined by arterial spin labeling: a validation study in isolated porcine kidneys. J Magn Reson Imaging 2007;26:353-358.

-6 Cutajar M, Thomas DL, Banks T, Clark CA, Golay X, Gordon I: Repeatability of renal arterial spin labelling MRI in healthy subjects. MAGMA 2012;25:145-153.

7 Gillis KA, McComb C, Foster JE, Taylor AH, Patel RK, Morris ST, Jardine AG, Schneider MP, Roditi GH, Delles C, Mark PB: Interstudy reproducibility of arterial spin labelling magnetic resonance imaging for measurement of renal perfusion in healthy volunteers at 3 tesla. BMC Nephrol 2014;15:23.
8 Michaely HJ, Schoenberg SO, Ittrich C, Dikow R, Bock M, Guenther M: Renal disease: value of functional magnetic resonance imaging with flow and perfusion measurements. Invest Radiol 2004;39:698-705.

-9 Rossi C, Artunc F, Martirosian P, Schlemmer HP, Schick F, Boss A: Histogram analysis of renal arterial spin labeling perfusion data reveals differences between volunteers and $\mathrm{pa}$ tients with mild chronic kidney disease. Invest Radiol 2012;47:490-496.

10 Lanzman RS, Wittsack HJ, Martirosian P, Zgoura P, Bilk P, Kröpil P, Schick F, Voiculescu A, Blondin D: Quantification of renal allograft perfusion using arterial spin labeling MRI: initial results. Eur Radiol 2010;20:14851491.

11 Heusch P, Wittsack HJ, Blondin D, Ljimani A, Nguyen-Quang $M$, Martirosian $P$, Zenginli H, Bilk P, Kröpil P, Heusner TA, Antoch G, Lanzman RS: Functional evaluation of transplanted kidneys using arterial spin labeling MRI. J Magn Reson Imaging 2014;40:84-89.

12 Artz NS, Sadowski EA, Wentland AL, Grist TM, Seo S, Djamali A, Fain SB: Arterial spin labeling MRI for assessment of perfusion in native and transplanted kidneys. Magn Reson Imaging 2011;29:74-82.

$\checkmark 13$ Levey AS, Stevens LA, Schmid CH, Zhang YL, Castro AF 3rd, Feldman HI, Kusek JW, Eggers P, Van Lente F, Greene T, Coresh J: A new equation to estimate glomerular filtration rate. Ann Intern Med 2009;150:604-612.

14 Xue H, Shah S, Greiser A, Guetter C, Chefdhotel C, Zuehlsdorff S, Guerhing J, Kellman P: Improved motion correction using image registration based on variational synthetic image estimation: application to inline T1 mapping of myocardium. J Cardiovasc Magn Reson 2011;13(suppl 1):P21.
15 Martirosian P, Klose U, Mader I, Schick F: FAIR true-FISP perfusion imaging of the kidneys. Magn Reson Med 2004;51:353-361.

16 Evangelidis GD, Psarakis EZ: Parametric image alignment using enhanced correlation coefficient maximization. IEEE Trans Pattern Anal Mach Intell 2008;30:1858-1865.

17 Schainuck LI, Striker GE, Cutler RE, Benditt EP: Structural-functional correlations in renal disease. II. The correlations. Hum Pathol 1970;1:631-641.

18 Bohle A, Mackensen-Haen S, von Gise H, Grund KE, Wehrmann M, Batz C, Bogenschütz $\mathrm{O}$, Schmitt H, Nagy J, Müller C, et al: The consequences of tubulo-interstitial changes for renal function in glomerulopathies. A morphometric and cytological analysis. Pathol Res Pract 1990;186:135-144.

19 Gardener AG, Francis ST: Multislice perfusion of the kidneys using parallel imaging: image acquisition and analysis strategies. Magn Reson Med 2010;63:1627-1636.

20 Cutajar M, Thomas DL, Hales PW, Banks T, Clark CA, Gordon I: Comparison of ASL and DCE MRI for the non-invasive measurement of renal blood flow: quantification and reproducibility. Eur Radiol 2014;24:1300-1308.

-21 Ritt M, Janka R, Schneider MP, Martirosian P, Hornegger J, Bautz W, Uder M, Schmieder RE: Measurement of kidney perfusion by magnetic resonance imaging: comparison of MRI with arterial spin labeling to para-aminohippuric acid plasma clearance in male subjects with metabolic syndrome. Nephrol Dial Transplant 2010;25:1126-1133.

22 Huang Y, Sadowski EA, Artz NS, Seo S, Djamali A, Grist TM, Fain SB: Measurement and comparison of $\mathrm{T} 1$ relaxation times in native and transplanted kidney cortex and medulla. J Magn Reson Imaging 2011;33:1241-1247.
Non-Contrast Renal MRI in Health and Disease
Nephron 2016;133:183-192 DOI: $10.1159 / 000447601$ 
23 Lee VS, Kaur M, Bokacheva L, Chen Q, Rusinek H, Thakur R, Moses D, Nazzaro C, Kramer EL: What causes diminished corticomedullary differentiation in renal insufficiency? J Magn Reson Imaging 2007;25:790-795.

24 Breidthardt T, Cox EF, Squire I, Odudu A, Omar NF, Eldehni MT, Francis ST, McIntyre CW: The pathophysiology of the chronic cardiorenal syndrome: a magnetic resonance imaging study. Eur Radiol 2015;25:1684-1691.

25 Fenchel M, Martirosian P, Langanke J, Giersch J, Miller S, Stauder NI, Kramer U, Claussen CD, Schick F: Perfusion MR imaging with FAIR true FISP spin labeling in patients with and without renal artery stenosis: initial experience. Radiology 2006;238:10131021.
26 Ott C, Janka R, Schmid A, Titze S, Ditting T, Sobotka PA, Veelken R, Uder M, Schmieder RE: Vascular and renal hemodynamic changes after renal denervation. Clin J Am Soc Nephrol 2013;8:1195-1201.

27 Schneider MP, Janka R, Ziegler T, Raff U, Ritt M, Ott C, Veelken R, Uder M, Schmieder RE: Reversibility of the effects of aliskiren in the renal versus systemic circulation. Clin J Am Soc Nephrol 2012;7:258-264.

28 Park SH, Wang DJ, Duong TQ: Balanced steady state free precession for arterial spin labeling MRI: initial experience for blood flow mapping in human brain, retina, and kidney. Magn Reson Imaging 2013;31:1044-1050.
29 Wang J, Zhang Y, Yang X, Wang X, Zhang J, Fang J, Jiang X: Hemodynamic effects of furosemide on renal perfusion as evaluated by ASL-MRI. Acad Radiol 2012;19:11941200.

30 Kiefer C, Schroth G, Gralla J, Diehm N, Baumgartner I, Husmann M: A feasibility study on model-based evaluation of kidney perfusion measured by means of FAIR prepared true-FISP arterial spin labeling (ASL) on a 3-T MR scanner. Acad Radiol 2009;16: 79-87.

31 Karger N, Biederer J, Lüsse S, Grimm J, Steffens J, Heller M, Glüer C: Quantitation of renal perfusion using arterial spin labeling with FAIR-UFLARE. Magn Reson Imaging 2000; 18:641-647. 